\title{
Rotational cooling of polar molecules by Stark-tuned cavity resonance
}

\author{
C. H. Raymond Ooi* \\ Fachbereich Physik der Universität Konstanz, Fach M674, D-78457 Konstanz, Germany \\ (Received 31 October 2002; revised manuscript received 17 April 2003; published 22 July 2003)
}

\begin{abstract}
A general scheme for rotational cooling of diatomic heteronuclear molecules is proposed. It uses a superconducting microwave cavity to enhance the spontaneous decay via Purcell effect. Rotational cooling can be induced by sequentially tuning each rotational transition to cavity resonance, starting from the highest transition level to the lowest one using an electric field. Electrostatic multipoles can be used to provide large confinement volume with essentially homogeneous background electric field.
\end{abstract}

DOI: 10.1103/PhysRevA.68.013410 PACS number(s): 33.80.Ps, 34.50.Ez, 32.60.+i, 42.55.Sa

\section{INTRODUCTION}

Currently, there are three main techniques to produce translationally cold molecules. The buffer gas [1] and Stark deceleration [2] cooling schemes employ trapping of the low field seekers, which requires the molecules to be in the appropriate internal states. The cold molecules produced from the photoassociation technique [4] are usually vibrationally hot, occupying a number of high-lying vibrational states. Vibrational cooling schemes using optimal control of ultrashort pulses have been proposed [5]. Translationally cold molecules are useful for precision spectroscopy and measurements, molecular optics and interferometry as well as for cold collisions studies, although they may be internally hot. It is also desirable to have translationally and internally cold molecules as well if the aim is to obtain molecular BoseEinstein condensate [3]. For the purpose of trapping, the molecules need to be cooled internally.

There has been no scheme to rotationally cool molecules although there are several methods of producing molecules in a single rotational state. A classic example is the electrostatic low- $J$ selector which has been used for the rotational state selection long ago [6]. Optical Stern-Gerlach effect has been demonstrated for atoms [7], and molecular state selection by lasers has been proposed [8]. However, these techniques are best applied to molecular beam. They are not cooling schemes since they do not employ a dissipative mechanism. Supersonic expansion is based on dissipation and may also produce rotationally cold molecules, but cannot be used to cool molecules which are already translationally cold since it requires high pressure and can only be applied mechanically on hot molecules. Besides, for translationally cold molecules it is more efficient to employ dissipative process for internal cooling which avoids the loss of molecules.

Recently, we have proposed one-dimensional (1D) translational cooling schemes for molecules which rely on a single optical spontaneous emission [9]. The schemes can be repeated for $3 \mathrm{D}$ cooling if the $1 \mathrm{D}$ cooled molecules can be brought back to the initial internal state again. The molecules in the excited rotvibrational states can redistribute significant amount of the internal energy into the translation motion by the state-changing inelastic collisions. At high temperature,

*Electronic address: ooi@spock.physik.uni-konstanz.de this becomes a problem for trapping due to the increased collision rate. Therefore, it is essential to remove the internal excitations by the spontaneous emissions fast enough before the inelastic collisions occur. The spontaneous emission carries away the entropy to the radiation, while the inelastic collision transfers entropy to the translational degree. For sufficiently dilute heteronuclear molecules, vibrational cooling occurs within $0.1-1 \mathrm{~s}$ time scale through infrared spontaneous emissions before the vibrational inelastic collisions take place. Thus, the vibrational entropy is discarded as radiation entropy instead of the translational entropy. However, the rotational spontaneous emissions can occur only in polar molecules and take much longer time, beyond the experimental time scale for dilute gas. For a dense gas, rotational decay may be enhanced through the many-body effect, but inelastic collision rate may become dominantly large.

In this paper, we propose a rotational cooling scheme for confined polar molecules. We use the external electric field to tune the internal transitions into resonance with a lossy microwave cavity (Fig. 1). This enhances the rotational spontaneous emissions via the Purcell effect [10], whereby the populations are transferred to a lower rotational level in stages and eventually to the ground rotational level. The Purcell effect has been experimentally demonstrated by Goy et al. using Rydberg atoms in a microwave cavity [11]. The

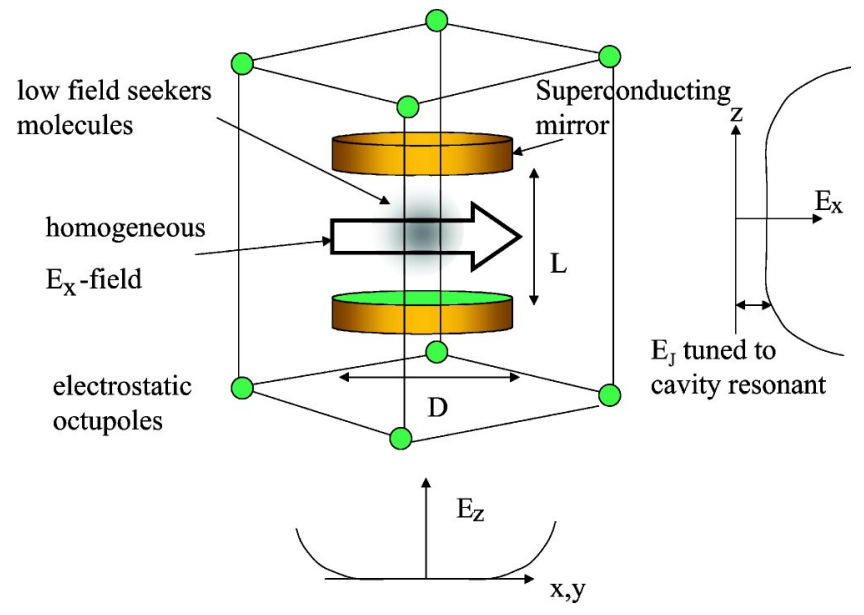

FIG. 1. Schematic of the apparatus for rotational cooling using cavity enhanced dissipation and electric field for resonance tuning and $3 \mathrm{D}$ confinement. 
cooling process requires long interaction time, and we have proposed a method to confine the molecules within the cavity field.

\section{INITIAL STATE}

We consider a gas of polar diatomic molecules at thermal equilibrium temperature $T$ with the Maxwell-Boltzmann distribution. We also assume that the gas is sufficiently dilute such that the inelastic collision is negligible throughout the cooling process. Below room temperature, the populations are essentially in the ground electronic state $|g, \Omega\rangle$. Thus, the population and the eigenenergy in the vibrational state $|n\rangle$ and the rotational states $|J, M\rangle$ with $J \geqslant \Omega$ (the total electronic angular momentum) are given, respectively, by

$$
\begin{aligned}
P(g, \Omega, n, J)= & P_{o}(2 J+1) \sum_{\Omega=1 / 2,3 / 2} \exp \left\{-E_{g, \Omega, n J} / k_{B} T\right\} \\
E_{g, n J, \Omega}= & h c\left\{T_{g}+\omega_{g}\left(n+\frac{1}{2}\right)-\omega_{g} x_{g}\left(n+\frac{1}{2}\right)^{2}\right. \\
& \left.+\left[B_{g}-\alpha_{g}\left(n+\frac{1}{2}\right)\right]\left[J(J+1)-\Omega^{2}\right]\right\},
\end{aligned}
$$

where $T_{g}, \omega_{g}, \omega_{g} x_{g}, B_{g}$, and $\alpha_{g}$ are the standard electronic, vibrational, and rotational constants (in wave number) [12].

From Eqs. (1) and (2), we estimate that for CsF molecule with $\left(\omega_{e}, \omega_{e} x_{e}, B_{e}, \alpha_{e}\right)=(352.56,1.61,0.184,0.0012) \mathrm{cm}^{-1}$ [12], only the first five rotational levels in the ground vibrational level $(n=0)$ are significantly occupied at a temperature around $1 \mathrm{~K}$. The rotational levels and the magnetic (Zeeman) states for cases $\Omega=0$ and $\Omega=\frac{1}{2}$ are shown in Figs. 2(a) and 3(a), respectively. Initially, the molecules are in all the magnetic states. In order to cool the molecules in all the magnetic states, the cavity must be able to support the linearly polarized as well as the circularly polarized photons corresponding to $\Delta M=0$ and \pm 1 transitions, respectively. If we assume that the cavity supports only the linearly polarized photons, only the states with $M \leqslant \pm \Omega$ will be sequentially Stark tuned to cavity frequency by an external static electric field for an enhanced spontaneous decay towards the ground level. The molecules in the other states (with $M>$ $\pm \Omega$ ) are off resonance with the cavity and their decays will not be enhanced. In this case, we will have to extract only the molecules in the states $M \leqslant \pm \Omega$ for rotational cooling.

\section{ROTATIONAL COOLING SCHEME}

In this section, we elaborate on the rotational cooling scheme based on the proposed setup apparatus (Fig. 1).

\section{A. Cooling mechanism}

The spacings of rotational levels in polar diatomic molecules increase monotonically with energy levels. We use this monotonic property to bring the populations from the high- $J$ levels to a low- $J$ (ground) level by enhanced rota-

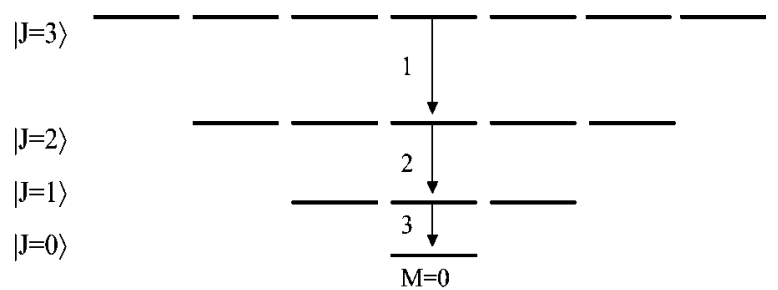

a)

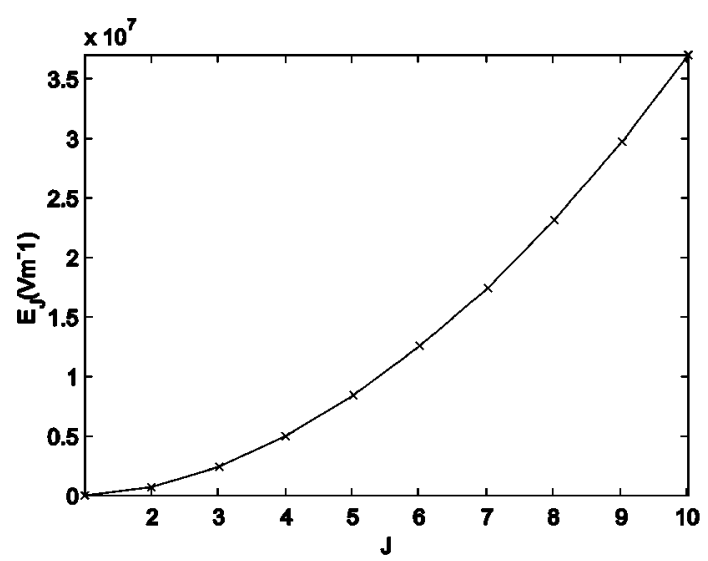

b)

FIG. 2. Spinless $(S=0)$ molecule such as CsF in state $\left|X^{1} \Sigma_{0}, n=0\right\rangle$ : (a) Rotational levels and the Zeeman states with a single ground state. Only the parallel transitions (1 to 3) with $\Delta M$ $=0$ are shown. (b) Electric fields for tuning the CsF molecules from the upper state $\left|J_{\max }=10, M=0\right\rangle$ to the lowest state $\mid J=0, M^{\prime}$ $=0\rangle$.

tional decay in a lossy microwave cavity. We want to bring each pair of transition $(|J, M\rangle$ to $|J-1, M-q\rangle$ with $q=0$, \pm 1 and $J \geqslant 1$ ) into resonance with the cavity each time, starting from the $J_{\max } \rightarrow J_{\max }-1$ transition and ending with the $J=\Omega+1 \rightarrow J=\Omega$ transition. We shall elaborate on the simplest scheme employing only the $\pi$ transitions $(q=\Delta M$ $=0$ ) [Figs. 2(a) and 3(a)], as well as the scheme which uses multiple polarized cavity for both the $\sigma$ and $\pi$ transitions.

\section{B. Cavity enhanced decay}

When the cavity is resonant with the radiative transition from $|J, M\rangle$ to $|J-1, M-q\rangle$, the decay rate is enhanced, given by the Purcell formula [13]

$$
\Gamma_{c, J, M, q}=\frac{2 \mu_{J, M, q}^{2}}{\varepsilon_{o} V \hbar} Q=\eta \Gamma_{o, J, M, q},
$$

where $\Gamma_{o, J, M, q}=16 \pi^{3} \mu_{J, M, q}^{2} / 3 \varepsilon_{o} h \lambda_{c}^{3}$ is the free space decay rate, $\eta=3 \lambda_{c}^{3} Q / 4 \pi^{2} V$ is the cavity enhancement factor, $\mu_{J, M, q}$ is the dipole transition matrix element, $\lambda_{J}$ is the transition wavelength resonant with the cavity frequency $\omega_{c}$ $=2 \pi c / \lambda_{c}, Q$ is the quality factor, and $V=\int\left|u_{m n}(\mathbf{r})\right|^{2} d^{3} r$ is the effective cavity volume for the Hermite-Gaussian $(m, n)$ transverse mode function $u_{m n}(\mathbf{r})$.

We consider a Gaussian mode in a symmetric confocal resonator (aligned along the $z$ axis, see Fig. 1), we have [14] 


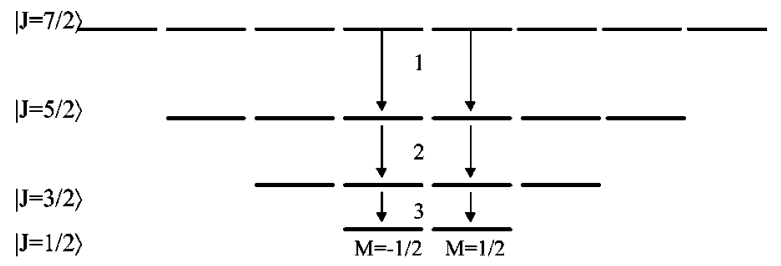

a)

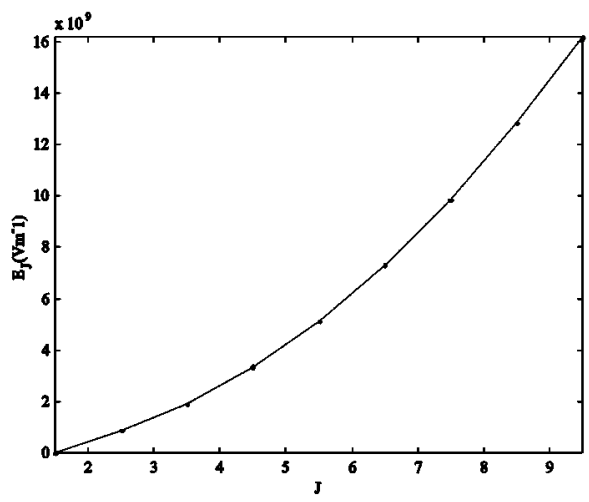

b)

FIG. 3. $\mathrm{OH}$ molecule in state $\left|X^{1} \Pi_{1 / 2}, n=0\right\rangle$ : (a) Rotational levels and the Zeeman states with two ground states. Six parallel transitions (1 to 3) are shown. (b) Electric fields for tuning the $\mathrm{OH}$ molecules from the upper states of $\left|J_{\max }=9.5, M= \pm 0.5\right\rangle$ to ground states $\left|J=0.5, M^{\prime}= \pm 0.5\right\rangle$.

the cavity spacing $L=\left(s+\frac{1}{2}\right) \lambda_{c} / 2$ for longitudinal-mode order $s$, field waist $w_{o}=\sqrt{\lambda_{c} L / 2 \pi}=\lambda_{c} \sqrt{\left(s+\frac{1}{2}\right) / 4 \pi}$, intensity $\left|u_{00}(\mathbf{r})\right|^{2}=\left[w_{o}^{2} / w^{2}(z)\right] e^{-2\left(x^{2}+y^{2}\right) / w^{2}(z)} \quad$ with $w^{2}(z)=w_{o}^{2}[1$ $\left.+\left(2 \lambda_{c} z / \pi w_{o}\right)^{2}\right]$. From these, the effective volume and the enhancement factor are rewritten as

$$
\begin{gathered}
V=L w_{o}^{2} \pi / 2=\frac{\lambda_{c} L^{2}}{4}=\left(s+\frac{1}{2}\right)^{2} \lambda_{c}^{3} / 16, \\
\eta=\frac{12 Q}{\pi^{2}\left(s+\frac{1}{2}\right)^{2}} .
\end{gathered}
$$

A large enhancement factor is obtained by using a superconducting cavity with large $Q$ and a lower-order mode $s$. The free and enhanced decay rates are dependent on $J$ and $M$ through the numerical factor in the matrix elements.

\section{Shielding thermal photons}

In the microwave regime, the mean number of thermal photons $\bar{n}\left(\omega_{c}\right)$ is not negligible. It enhances the spontaneous decay rate as $\Gamma_{o, J, M, q}=\left(\mu_{J, M, q}^{2} \omega_{c}^{3} / 3 \varepsilon_{o} \hbar \pi c^{3}\right)\left[2 \bar{n}\left(\omega_{c}\right)+1\right]$. However, it reduces the cooling efficiency since the decaying states cannot be completely depopulated due to the simultaneous incoherent excitations of the thermal photons. The usual way to reduce the thermal photons is by cooling of the apparatus. The inner surface of the apparatus is coated with a microwave photon absorbing material such as graphite, while the outer surface can also be shielded with a photonic band-gap structure [15]. Thus, we can set $\bar{n}=0$ and write the free space decay rate for $\pi$ transition $|J, M\rangle \rightarrow|J-1, M\rangle$ and $\sigma$ transition $|J, M\rangle \rightarrow|J-1, M \mp 1\rangle[16]$, respectively, as

$$
\Gamma_{o, J, M, q}=\frac{\|\mu\|^{2}}{\lambda_{c}^{3}} g(J, M) 2.8187 \times 10^{46} \text { (in S.I.), }
$$

where $\|\mu\|$ is the reduced electric dipole matrix element for the vibronic state, and $g(J, M)=[(J+M)(J-M)] /[(2 J$ $-1)(2 J+1)]$ and $[(J \pm M+1)(J \pm M+2)] /[(2 J-1)(2 J$ $+1)$ ] for the $\pi$ transition and $\sigma$ transition, respectively.

\section{Electric-field tuning}

In order to enhance the decay of each transition, we can either tune the cavity or the levels into resonance with each other. In principle, we can construct a tunable cavity by changing the distance between the mirrors. First, the molecules are put into the cavity tuned to enhance the spontaneous emission of the highest rotational state, from $J_{\max }$ to $J_{\max }-1$. Then, the cavity is tuned to the next lower transition and so on until all the populations are brought to a single rovibrational state $J=0$. Here, $L$ changes from ( $s$ $\left.+\frac{1}{4}\right) \lambda_{J_{\max }} / 2$ to $\left(s+\frac{1}{4}\right) \lambda_{\Omega} / 2$. However, this approach is not very feasible in practice [17].

It is more realistic to tune the transition levels into resonance with cavity frequency using an external electric field. For $\Delta M=0$ transitions, photons are most likely to be emitted perpendicular to the dipole. To maximize the molecular dipole-cavity field coupling strength and the spontaneous decay rate, the dipole should be parallel to the cavity electric field (in $x-y$ plane). Therefore, the tuning electric field is applied along the $x$ axis so that it aligns the dipole along the cavity field (see Fig. 1). Here, the $\Delta M= \pm 1$ transitions can be enhanced too as the photons are emitted in all directions, although the most probable direction is parallel to the dipole. If the tuning electric field is along the cavity axis, the $\Delta M$ $=0$ transition may not be optimally enhanced because no photon is emitted exactly along the dipole. However, there is still some enhancement from the off-axis modes because the microwave cavity is essentially a closed cavity which provides a large solid angle of mode confinement.

A pair of closely spaced electrostatic plates can be introduced into the cavity to produce a tunable homogeneous transverse electric field $E_{J}$ perpendicular to the cavity axis (say along the $x$ direction) for Stark tuning. Thus, the edges of the superconducting cavity mirrors can be shielded from the strong electric field using a metallic casing or coating to maintain a constant high- $Q$ value.

The energy separation between states $|J, M\rangle$ and $\mid J$ $-1, M-q\rangle$ in the electric field $E_{J, M, q}$ is

$$
\begin{aligned}
\Delta \mathcal{E}_{n, J, M, q}= & h c 2 J\left\{B_{e}-\alpha_{e}\left(n+\frac{1}{2}\right)\right\} \\
& +\frac{\left(\mu E_{J, M, q}\right)^{2}}{2 h c B_{e}}\left(f_{J, M}-f_{J-1, M-q}\right),
\end{aligned}
$$




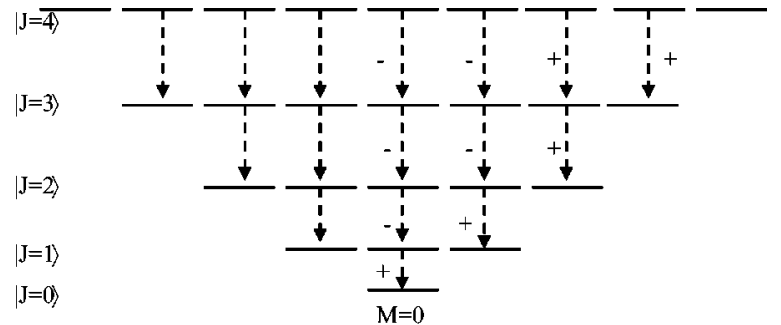

a)
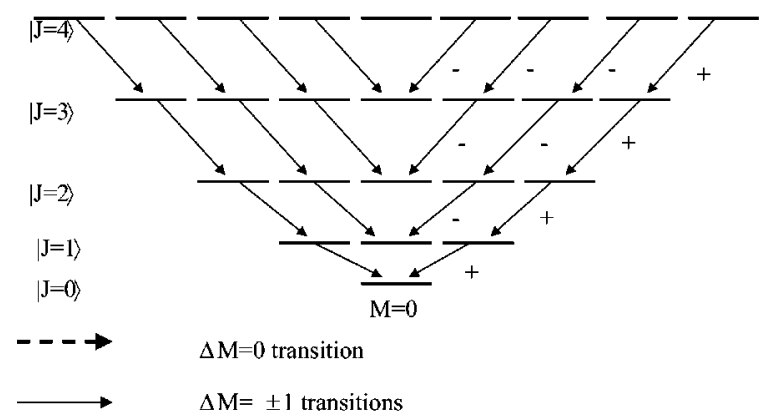

b)

FIG. 4. The signs of $f_{J, M^{\prime}}-f_{J-1, M}$ in Eq. (8) for (a) $\pi$ transitions $(\Delta M=0)$ and (b) $\pi$ transitions $(\Delta M= \pm 1)$. Due to the quadratic dependence of the Stark shift on $M$, the transitions $|J| M \mid$, $+1\rangle \rightarrow|J-1| M|\rangle$, are symmetrical to $|J,-| M|-1\rangle \rightarrow \mid J-1$, $-|M|\rangle$.

where

$$
\begin{aligned}
f_{J, M}-f_{J-1, M-q}= & \frac{J(J+1)-3 M^{2}}{J(J+1)(2 J-1)(2 J+3)} \\
& -\frac{J(J-1)-3(M-q)^{2}}{(J-1) J(2 J-3)(2 J+1)}
\end{aligned}
$$

and $\mu$ is the electric dipole moment.

From Eq. (7), it is possible to keep a pair of transition at cavity resonance $\Delta \mathcal{E}_{n, J, M, q}=h c / \lambda_{c}$ by varying the electric field for each set of $J, M, q$. Setting $n=0$, the required electric field for each transition can be predicted as

$$
E_{J, M, q}=\frac{h c}{\mu} \sqrt{\frac{2 B_{e}}{f_{J, M}-f_{J-1, M-q}}\left\{\frac{1}{\lambda_{c}}-J\left(2 B_{e}-\alpha_{e}\right)\right\}} .
$$

We need to fix the cavity parameters through $\lambda_{c}$ such that the argument in the square root is positive. In general, $f_{J, M}$ $-f_{J-1, M-q}$ can be positive or negative depending on the transition, so it may not be possible to use a single value of $\lambda_{c}$ to tune all transitions to cavity resonance. It is only possible to tune those transitions with negative values of $f_{J, M}$ $-f_{J-1, M-q}$ [the "negative sign" transitions in Figs. 4(a) and $4(\mathrm{~b})]$ to cavity resonance by choosing the cavity parameter $\lambda_{c}$ which gives a negative nominator in Eq. (8), $1 / \lambda_{c}$ $<J\left(2 B_{e}-\alpha_{e}\right)$ or $L>s+\frac{1}{2} / 2 J\left(2 B_{e}-\alpha_{e}\right)$, in order to obtain real values of $E_{J, M, q}$. This can be satisfied for $J \geqslant 1$ and

$$
J>\left\{\frac{1}{2}\left[1+6 M^{2}+\sqrt{\left(1+3 M^{2}+36 M^{4}\right)}\right]\right\}^{1 / 2},
$$

if we set

$$
\frac{1}{\lambda_{c}}=\left(2 B_{e}-\alpha_{e}\right) \text { or } L=\frac{s+\frac{1}{2}}{2\left(2 B_{e}-\alpha_{e}\right)},
$$

and Eqs. (6) and (8) become

$$
\begin{gathered}
\Gamma_{o, J, M, q}=\|\mu\|^{2}\left(2 B_{e}-\alpha_{e}\right)^{3} g(J, M) 2.8187 \times 10^{46} \\
E_{J, M, q}=\frac{h c}{\mu} \sqrt{\frac{2 B_{e}\left(2 B_{e}-\alpha_{e}\right)}{f_{J, M}-f_{J-1, M-q}}(1-J)}
\end{gathered}
$$

Condition (9) applies to the $\pi$ transitions [Fig. 4(a)] and to the $\sigma$ transitions [Fig. 4(b)], where $f_{J, M}-f_{J-1, M-q}$ are negative. It also applies to $|J=1, M=0\rangle \rightarrow|0,0\rangle$ although $f_{1,0}-f_{0,0}$ is positive [Fig. 4(a)] because Eq. (11) vanishes for $J=1$.

On the other hand, the "corner" $\pi$ transitions $\mid J, M$ $= \pm J\rangle \rightarrow|J-1, \pm(J-1)\rangle$ have a positive denominator,

$$
f_{J, \pm J}-f_{J-1, \pm(J-1)}=\frac{4 J+3}{(2 J+1)(2 J+3) J(J+1)} .
$$

The cavity value $\lambda_{c}$ defined by Eq. (9) gives a negative nominator and cannot be used to tune these transitions. The "corner" transitions and those $\pi$ transitions [Fig. 4(a)] with "positive sign" can be tuned for cooling by using a different cavity parameter which satisfies a positive numerator in Eq. (8), $1 / \lambda_{c}>J\left(2 B_{e}-\alpha_{e}\right)$. A reasonable choice is

$$
\frac{1}{\lambda_{c}}=J_{\max }\left(2 B_{e}-\alpha_{e}\right)
$$

and we have

$$
\begin{aligned}
\Gamma_{o, J, M, q}= & J_{\max }^{3}\|\mu\|^{2}\left(2 B_{e}-\alpha_{e}\right)^{3} g(J, M) 2.8187 \\
& \left.\times 10^{46} \text { (in S.I. }\right), \\
E_{J, M, q}= & \frac{h c}{\mu} \sqrt{\frac{2 B_{e}\left(2 B_{e}-\alpha_{e}\right)}{f_{J, M}-f_{J-1, M-q}}\left\{J_{\max }-J\right\} .}
\end{aligned}
$$

If the cavity only supports the linearly polarized photons, only the parallel or the $\pi$ transitions $(\Delta M=0)$ can be enhanced. For the moment, we consider only the $\pi$ transitions from the states $|J| M,|\leqslant \Omega\rangle$ to $|J-1| M,|\leqslant \Omega\rangle$. The transitions are tuned to cavity resonance each time using the electric field, while all other Zeeman states are not in resonance with the cavity due to the different Stark shifts. Only those molecules in the states $|J,-\Omega \leqslant M \leqslant \Omega\rangle$ are rotationally cooled down to a number of $2 \Omega+1$ states in the ground level, $|\Omega,-\Omega \leqslant M \leqslant \Omega\rangle$. For example, with $\mathrm{OH}$ molecules in state ${ }^{2} \Pi_{\Omega=1 / 2}$ [Fig. 3(a)], rotational cooling leads to two internal ground states. For spinless molecules such as CsF in the ground electronic state $X^{1} \Sigma_{\Omega=0}$ [Fig. 2(a)], the molecules can be cooled to a single internal state $|0,0\rangle$. The number of tuning steps required for the $\Delta M=0$ transitions is $(\Omega+1)\left(J_{\max }-\Omega\right)$ for integer $\Omega$, and $\left(\Omega+\frac{1}{2}\right)\left(J_{\max }-\Omega\right)$ for 


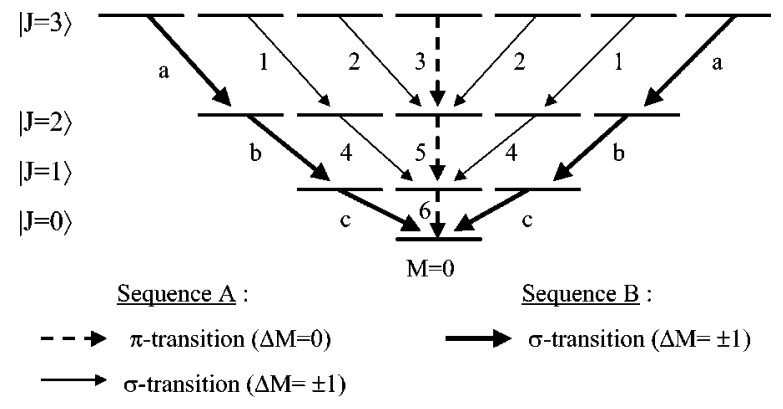

FIG. 5. A scheme of the tuning sequence for rotational cooling using a multiple field polarization cavity. Sequence $A$ (transition 1 to transition 6) is used with the cavity which satisfies $1 / \lambda_{c}=\left(2 B_{e}\right.$ $-\alpha_{e}$ ). Sequence $B$ is composed of the "corner transitions" ( $a$ to $c$ ) for the cavity with $1 / \lambda_{c}=J_{\max }\left(2 B_{e}-\alpha_{e}\right)$.

half integer $\Omega$. The tuning electric fields calculated from Eq. (11) are shown in Figs. 2(b) and 3(b) for different $J$ of the decaying state.

From Figs. 4(a) and 4(b), we see that it is possible establish a scheme to cool the molecules in most states by using a multiple field polarization cavity which can support all the three polarized photons (linear, $\sigma^{-}$, and $\sigma^{+}$) corresponding to $\Delta M=0, \pm 1$ transitions, respectively. This enables the molecules in all the Zeeman states, except the "corner states" $|J, \pm J\rangle$, to be Stark tuned for cooling into a single ground level (or single internal state for case $\Omega=0$ ). Due to the quadratic dependence of the Stark shift on $M$, two pairs of states can be Stark tuned to cavity resonance each time; but only one pair of states for $M=0 \Leftrightarrow M=0$ transition.

The total number of steps for cooling with multiple polarizations is generally given by $\frac{1}{2}\left(J_{\max }-\Omega\right)\left(J_{\max }+\Omega+3\right)$ for integer $\Omega$ and $\frac{1}{2}\left(J_{\max }-\Omega\right)\left(J_{\max }+\Omega+2\right)$ for half integer $\Omega$, while the total number of transitions is $J_{\max }\left(J_{\max }+2\right)-\Omega(\Omega$ $+2)$. The possible sequence of tuning is shown in Fig. 5 for the molecules with spin $S=0$. The two sequences $A$ and $B$ correspond to different cavity parameters given by Eqs. (9) and (12), respectively. Sequence $A$ requires $\frac{1}{2}\left(J_{\max }\right.$ $-\Omega)\left(J_{\max }+\Omega+1\right)$ steps for integer $\Omega$ and $\frac{1}{2}\left(J_{\max }^{2}-\Omega^{2}\right)$ steps for half integer $\Omega$, while sequence $B$ requires $\left(J_{\max }-\Omega\right)$ steps. Sequence $A$ cools $J_{\max }+\Omega+1$ times more states than sequence $B$, but the transitions of both the sequences are complementary. The tuning electric fields for sequences $A$ and $B$ are calculated from Eqs. (11) and (14) as shown in Figs. 6(a) and 6(b), respectively.

\section{E. Doppler broadening}

The cavity linewidth $\kappa=\omega_{c} / Q$ must be larger than the maximum Doppler shift in order for the moving molecules to stay in resonance with the cavity $\omega_{c} / Q>\omega_{D}=\omega_{c} P_{\max } / M c$. For thermal ensemble of $\mathrm{OH}$ molecules at about $10 \mathrm{~K}\left(70 \mathrm{~ms}^{-1}\right)$, the maximum quality factor we can use is $Q \approx M c / P_{\max } \approx 10^{6}$. This value just gives the highest enhancement factor in the bad cavity regime. So, it is not helpful to use larger $Q$. The momentum distribution of the molecules is essentially unaffected by the small microwave photon recoil momentum from rotational spontaneous emissions unless the molecules are ultracold.

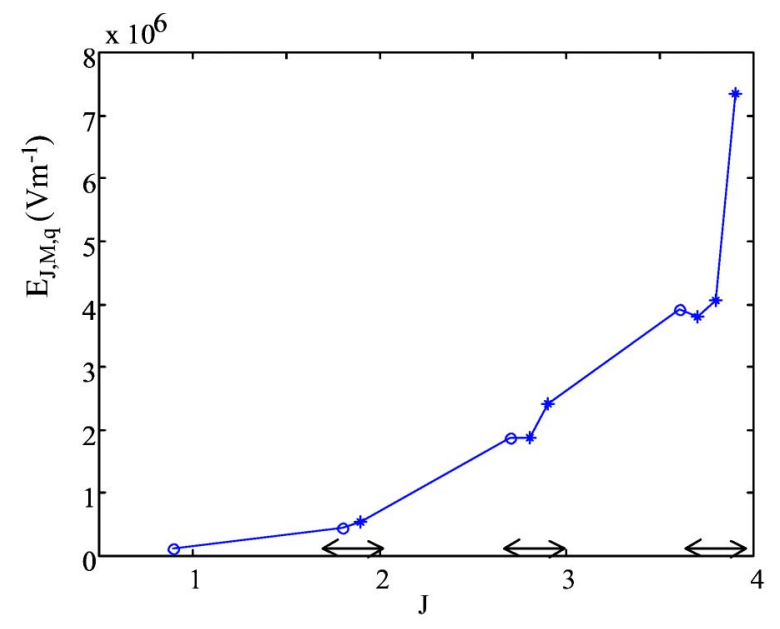

a)

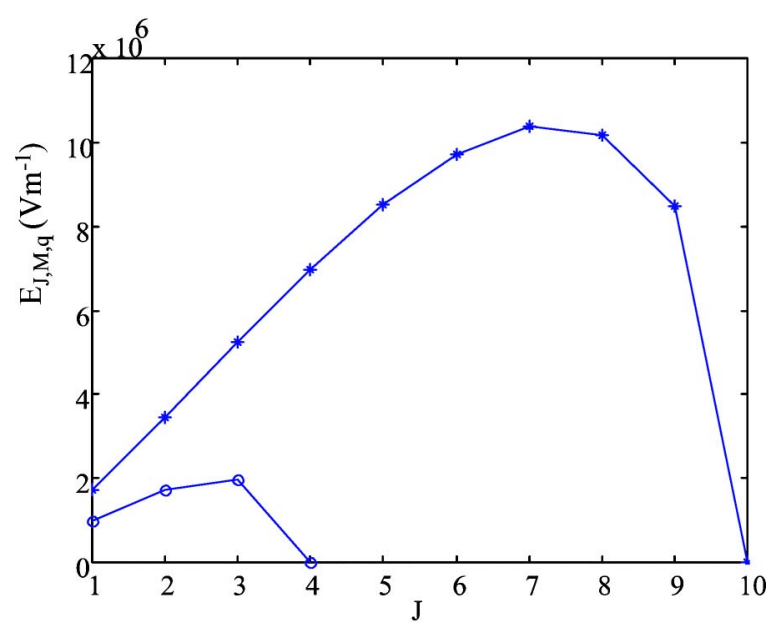

b)

FIG. 6. Electric fields for tuning the CsF molecules using multiple field polarization cavity. (a) Sequence $A$-The points corresponding to each $J$ are displaced slightly from each other for clarity and connected by a line to show the tuning sequence, which starts from the largest electric-field value. The points " $o$ " correspond to the $\pi$ transitions. (b) Sequence $B-$ The " $o$ " line is for $J_{\max }=4$, while the "*" line is for $J_{\max }=10$. The abscissa $J$ labels the upper rotational state for each transition.

\section{F. Confinement}

The maximum diameter (radial dimension) for the confocal cavity geometry can be estimated as $D=\sqrt{3} L$ (see Fig. 1). For a typical microwave cavity dimension of $1 \mathrm{~mm}$ and molecules with velocity of say $70 \mathrm{~ms}^{-1}$, the interaction time of $\Delta t=w / u=0.01 \mathrm{~ms}$ is much shorter than the enhanced lifetime. Therefore, we need to confine the molecules within the cavity.

Figure 1 shows the proposed rotational cooling apparatus with the cavity and electric octupoles. The higher-order electrostatic poles can be used to confine the low field seekers with kinetic energy below about $1 \mathrm{~K}$ [18]. This background field also prevents the Majorana flop into untrapping state.

It may be possible to confine the molecules using a nonstick solid material such as teflon which has low surface 
adhesion to certain molecules. However, this is only applicable to a gas at room temperature and not to translationally cold gas because the collisions with the solid wall will lead to translational thermalization. Since the molecules in the solid material do not rotate and have no angular momentum, collisions with the surface do not cause rotational transition via conservation of angular momentum. The main advantage of the solid material confinement is, of course, the infinitely long trapping time.

\section{G. Numerical estimates}

We estimate the cooling time with the typical applied tuning field for two types of molecules only for the $\pi$ transitions involving the middle states $(-\Omega \leqslant M \leqslant \Omega)$. First, we consider $\mathrm{CsF}$ molecules in state $X^{1} \Sigma$ [Fig. 2(a)] with $B_{e}$ $=0.1844 \mathrm{~cm}^{-1}$ and $\alpha_{e}=1.18 \times 10^{-3} \mathrm{~cm}^{-1}$, and the electric dipole moment $\mu=7.87 \mathrm{D}$ [21]. The required electric field of in the order of $10^{7} \mathrm{~V} \mathrm{~m}^{-1}$ [Fig. 2(b)]. The cavity spacing from Eq. (9) is $L=2.04 \mathrm{~cm}$ for mode order $s=1$. The cavity diameter is $D \sim 3.5 \mathrm{~cm}$. If the distance between the opposite electrostatic poles, which produces the homogeneous electric field $E_{J}$, is $1 \mathrm{~cm}$, the required voltage is around $100 \mathrm{kV}$. The free space decay rate is $\Gamma_{o, J, 0,0}=\left[J^{2} /(2 J-1)(2 J+1)\right] 9.65$ $\times 10^{-7} \mathrm{~s}^{-1}$ and, for $Q=10^{6}$, the enhancement factor of $\eta$ $=0.54 \times 10^{6}$ boost the decay rate to $\Gamma_{c, J, 0,0}=0.52\left[J^{2} /(2 J\right.$ $-1)(2 J+1)] \mathrm{s}^{-1}$. Supposing that a duration of $t_{J}$ $=4 / \Gamma_{c, J, 0,0} \approx 8\left[(2 J-1)(2 J+1) / J^{2}\right]$ is allocated for each stage of decay from level $J$, then the total duration for enchanced decay from $J_{\max }=5$ to $J_{o}=0$ is

$$
\begin{aligned}
t_{\text {tot }} & =\sum_{J=J_{o}+1}^{J_{\max }} t_{J} \\
& =\sum_{J=1}^{5} 8 \frac{(2 J-1)(2 J+1)}{J^{2}} \approx 150 \mathrm{~s}(\sim 2.5 \mathrm{~min}) .
\end{aligned}
$$

Since the trapping volume is macroscopically large, many molecules can be confined, and this allows the use of sufficiently dilute gas to reduce the loss rate of molecules due to state-changing collisions. Due to the absence of Majorana flop, a significant number of molecules should remain confined for long enough time for the rotational cooling to take place.

Next, we estimate the essential cooling parameters using $\mathrm{OH}$ molecules [with the levels in Fig. 3(a)], which have a large rotational constants $B_{e}=18.91 \mathrm{~cm}^{-1}$ and $\alpha_{e}$
$=0.724 \mathrm{~cm}^{-1}$ [19], and the static dipole moment of $\mu$ $=1.6676 \mathrm{D}[20]$. From Fig. 3(b), we find that the required electric fields are around $10^{10} \mathrm{~V}=\mathrm{m}^{-1}$. Even for cavity dimension of $0.1 \mathrm{~mm}$, the required voltage is very large, $V$ $\sim 1000 \mathrm{kV}$ and the free space decay rate is $\Gamma_{o, J, M, 0}=[(J$ $+1 / 2)(J-1 / 2)] /[(2 J-1)(2 J+1)] 4.4521 \times 10^{-2} \mathrm{~s}^{-1}$. For a small $Q=10^{3}$ and assuming $J_{\max }=5$, we have

$$
\eta=\frac{16000}{3 \times(3.14)^{2}}=540.93
$$

and

$$
t_{t o t}=\sum_{J=1}^{5} 4 \Gamma_{c, J, M, 0}^{-1}=\sum_{J=1}^{5} 4 \frac{2(2 J-1)(2 J+1)}{24(J+1 / 2)(J-1 / 2)} \approx 6.7 \mathrm{~s} .
$$

For $\mathrm{OH}$, the required electric field may be too large to be realized in practice, and it is due to the unusually large rotational constant $B_{e}$. However, it requires a small cavity $Q$ and the cooling time can be considerably shortened if the highest $Q$ in the bad cavity regime is used.

\section{CONCLUSIONS}

We have proposed a rotational cooling scheme for polar molecules using the Stark-tuned internal levels in a superconducting microwave cavity. A homogeneous electric field is applied for Stark tuning each transition to cavity resonance to enhanced rotational spontaneous emissions. Sequential tuning and the use of the cavity, which supports multiple field polarizations, enable all the molecules to be cooled towards the ground rotational level. The numerical estimates for $\mathrm{CsF}$ molecules show that the scheme requires high voltage which can be realized using the state-of-the-art technology. However, the molecules with unusually large rotational constants require electric field beyond the current capability. The typical cooling time of 1 min with a large- $Q$ enhancement in a dissipative cavity regime can be realized for most molecules with moderate rotational constant and large dipole moment.

\section{ACKNOWLEDGMENTS}

We gratefully acknowledge support from the Deutsche Forschungsgemeinschaft (Forschergruppe Quantengase) and Optik Zentrum Konstanz. We also thank Dr. Pepijn Pinkse and Professor Rempe for hospitality at MPQ, and Dr. MacAdam for introducing the Stark ball device.
[1] J.D. Weinstein, R. deCarvalho, T. Guillet, B. Friedrich, and J. Doyle, Nature (London) 395, 148 (1998).

[2] Hendrick L. Bethlem, Giel Berden, Floris M.H. Crompvoets, Rienk T. Jongma, J.A. van Roij, and Gerard Meijer, Nature (London) 406, 491 (2000).

[3] P. Schewe, J. Riordon, and B. Stein, Phys. News Update 581, 1 (2002).

[4] Y.B. Band and P.S. Julienne, Phys. Rev. A 51, R4317 (1995).
[5] S.G. Schirmer, Phys. Rev. A 63, 013407 (2001).

[6] H.K. Hughes, Phys. Rev. 72, 614 (1947).

[7] T. Sleator et al., Phys. Rev. Lett. 68, 1996 (1992).

[8] P. Domokos, T. Kiss, and J. Janszky, Eur. Phys. J. D 14, 49 (2001).

[9] C.H. Raymond Ooi, Karl-Peter Marzlin, and Jürgen Audretsch, Eur. Phys. J. D 22, 259 (2002).

[10] E.M. Purcell, Phys. Rev. 69, 681 (1946). 
[11] P. Goy, J.M. Raimond, M. Cross, and S. Haroche, Phys. Rev. Lett. 50, 1903 (1983).

[12] G. Herzberg, Molecular Spectra and Molecular Structure I. Spectra of Diatomic Molecules (Van Nostrand, Toronto, 1950).

[13] Marlan O. Scully and Muhammad Suhail Zubairy, Quantum Optics (Cambridge University Press, Cambridge, 1997).

[14] Amnon Yariv, Quantum Electronics (Wiley, New York, 1989).

[15] Christopher M. Cornelius and Jonathan P. Dowling, Phys. Rev. A 59, 4736 (1999).

[16] Harold W. Kroto, Molecular Rotation Spectra (Wiley, London, 1975).

[17] Pepijn Pinkse (private communication).

[18] A Stark ball device has been developed [K.B. MacAdam and
C.S. Hwang, Rev. Sci. Instrum. 74, 2267 (2003)]. The device can provide confinement for the molecules in three dimensions in a large volume of homogeneous or zero electric field. This device is ideal for our purpose. It uses 16 electrostatic rods organized in spherical symmetry and with versatile electronics control of electric field profiles.

[19] Aleksandr A. Radzig and Boris M. Smirnov, Reference Data on Atoms, Molecules and Ions, Springer Series in Chemical Physics Vol. 31 (Springer-Verlag, Berlin, 1985).

[20] W.L. Meerts and A. Dymanus, Chem. Phys. Lett. 23, 45 (1973).

[21] Robert M. Hill and Thomas F. Gallagher, Phys. Rev. A 12, 451 (1975). 